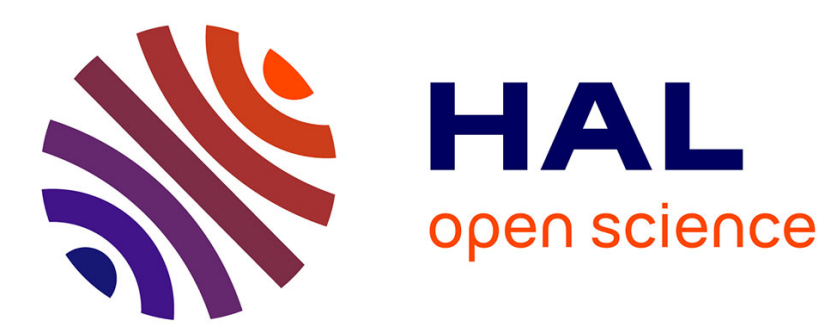

\title{
Effet de la dilatation thermique sur la valeur de la constante de curie des ferrites
}

Louis Néel

\section{To cite this version:}

Louis Néel. Effet de la dilatation thermique sur la valeur de la constante de curie des ferrites. J. Phys. Radium, 1951, 12 (3), pp.258-259. 10.1051/jphysrad:01951001203025800 . jpa-00234379

\section{HAL Id: jpa-00234379 https://hal.science/jpa-00234379}

Submitted on 1 Jan 1951

HAL is a multi-disciplinary open access archive for the deposit and dissemination of scientific research documents, whether they are published or not. The documents may come from teaching and research institutions in France or abroad, or from public or private research centers.
L'archive ouverte pluridisciplinaire HAL, est destinée au dépôt et à la diffusion de documents scientifiques de niveau recherche, publiés ou non, émanant des établissements d'enseignement et de recherche français ou étrangers, des laboratoires publics ou privés. 


\title{
EFFET DE LA DILATATION THERMIQUE SUR LA VALEUR DE LA GONSTANTE DE GURIE DES FERRITES
}

\author{
Par Louis NÉEL.
}

\begin{abstract}
Sommaire. - L'auteur montre que l'existence de fortes anomalies de dilatation dans les ferrites entraîne nécessairement une variation thermique importante des coefficients de champ moléculaire. Il en résulte que la constante de Curie asymptotique doit avoir une valeur plus grande que la valeur théorique classique. Pour la magnétite, l'augmentation observée est très voisine de la valenr calculéc d'après les données dilatométriques.
\end{abstract}

Il ressort d'études récentes, dues à A Serres [1] et à P. Maroni [2], que l'inverse $\frac{\mathrm{I}}{\chi_{M}}$ de la susceptibilité magnétique moléculaire des ferrites varie au-dessus de leur point de Curie, en fonction de la température absolue $T$, suivant la loi

$$
\frac{\mathrm{I}}{\chi_{1}}=\frac{T}{C}+\frac{\mathrm{I}}{\%_{0}}-\frac{\sigma}{T-1}
$$

qui résulte de la théorie du ferrimagnétisme [3], pourvu que $T$ ne soit pas trop proche du point de Curie ferromagnétique. Le Tableau donne les valeurs correspondantes de $C$ pour quelques ferrites ainsi que pour $\mathrm{Fe}_{2} \mathrm{O}_{3} \alpha$, substance antiferromagnétique.

\section{Tableau.}

\begin{tabular}{|c|c|c|c|c|c|}
\hline & & & 1 & & $\begin{array}{l}\text { Autenr } \\
\text { des }\end{array}$ \\
\hline Substance. & $\because$ & $1^{\prime}$. & $x_{0}^{\prime}$ & $-\gamma .10^{5}$. & mesures. \\
\hline $\mathrm{Fe}_{2} \mathrm{O}_{3}, \mathrm{Ig}_{\mathrm{g}} \mathrm{O} \ldots$ & I多, 0 & 8,8 & $\mathbf{1} 53$ & 2,76 & [1] \\
\hline $\mathrm{Fe}_{2} \mathrm{O}_{3} \mathrm{NiO} \ldots$ & 19,5 & IO, 0 & I 86 & 2,77 & {$[2]$} \\
\hline $\mathrm{Fe}_{2} \mathrm{O}_{3} \mathrm{CoO} \ldots$ & І 3,9 & II, 8 & 109 & $\mathrm{I}, \mathrm{I} 7$ & [2] \\
\hline $\mathrm{Fe}_{2} \mathrm{O}_{3} \mathrm{FeO} O$ & If, 2 & I I , 9 & 98 & 1,37 & [2] \\
\hline $\mathrm{Fe}_{2} \mathrm{O}_{3} \ldots \ldots$ & I 3,6 & 8,8 & $3 \Upsilon 6$ & I, 27 & [4] \\
\hline
\end{tabular}

La théorie indique que $C$ devrait être égal [3] [5] à la somme $C^{\prime}$, donnée dans le Tableau, des valeurs habituelles des constantes de Curie atomiques des ions correspondants dans les sels dont le point de Curie est voisin du zéro absolu. On s'aperçoit au contraire que $C$ est notablement plus grand que $C^{\prime}$.

Comme nous l'avons suggéré antérieurement [5], il est possible que cette discordance provienne de la variation thermique du champ moléculaire dont nous avons jadis souligné l'importance [6]. Supposons pour simplifier que seul, parmi les trois coefficients qui caractérisent le champ moléculaire des ferrites [7], le coefficient $n$ soit une fonction de $T$, $\alpha$ et $\beta$ restant constants, et posons

$$
n=n_{0}(\mathrm{I}+\gamma \boldsymbol{T}) .
$$

Dans ces conditions, l'expression de la variation thermique de la susceptibilité conserve la forme (I), mais la signification des coefficients change : en particulier, la constante $C$ est liée à la constante de Curie théorique $C^{\prime}$ par la relation

$$
\frac{\mathrm{r}}{C}=\frac{\mathrm{r}}{C^{\prime}}+\frac{\gamma}{\gamma_{0}} .
$$

Cette formule a permis de calculer les valeurs de $\gamma$ données dans le Tableau : elles varient de - r,2 à $-2,8 \cdot \mathrm{IO}^{-4}$. C'est un ordre de grandeur raisonnable, car, si nous attribuons la diminution du champ moléculaire à l'augmentation progressive de la distance $d$ séparant deux atomes voisins à la suite de la dilatation thermique, on trouve que, le coefficient de dilatation linéaire $\lambda$ étant de $16.10^{-6}$ (au voisinage de $\mathrm{rooo}^{\circ} \mathrm{K}$ ), $n$ varie comme $d^{-p}$ où $p$ vaut de 7,3 à i 7,3 selon le corps. Cette décroissance est comparable à celle des forces de répulsion interatomiques, où $p$ est compris entre 8 et i 4 [8].

Cette variation thermique de $n$ est accompagnée d'une anomalie de longueur au zéro absolu. En effet, lorsqu'une substance magnétiquement saturée subit une variation relative $r$ de longueur, l'énergie $E$ d'échange, par molécule-gramme, s'écrit

$$
E=E_{0}\left(\mathrm{I}+\frac{\dddot{r} r}{i}\right) \cdot
$$

En passant de l'état des spins ordonnés à l'état désordonné une telle substance subit [6] une variation relative $r_{0}$ de longueur donnée par

$$
r_{0}=\frac{E_{0} k_{0} \gamma}{9 V \lambda},
$$

où $V$ est le volume atomique et $k_{0}$ le coefficient de compressibilité cubique. Pour la magnétite, 
$V^{\top}=45 \mathrm{~cm}^{3}, k_{0}=-0,54.1 \mathrm{o}^{-12}, \lambda=\mathrm{i} 6 . \mathrm{Io}^{-6}$, tandis que les données de Maroni permettent de calculer $E_{0}=2,55 . \mathrm{Io}^{11} \mathrm{ergs}:$ on trouve alors $r_{0}=2,9 \cdot \mathrm{IO}^{-3}$. Or, il résulte des expériences dilatométriques de Chevenard [9] que l'anomalie de longueur, extrapolée au zéro absolu, vaut $3,0 . \mathrm{Io}^{-3}$. Cet accord excellent n'est sans doute qu'accidentel : pour les ferrites de magnésium et de nickel, l'anomalie de longueur observée est plus petite que la valeur calculée. Cela montre sans doute que le problème a été trop simplifié : d'une part en effet, on a négligé les termes d'oscillation [6], d'autre part, on a attribué le même coefficient $\gamma$ de variation thermique aux trois champs moléculaires caractéristiques des ferrites.

Signalons que Pauthenet [10] a calculé la variation thermique de l'aimantation spontanée des ferrites de Fe, Co, Ni et trouvé des valeurs en accord avec l'expérience, en utilisant les valeurs de $n_{0}, \alpha$ et $\beta$, corrigées de la dilatation, obtenues par Maroni d'après les expériences faites dans la région paramagnétique.

En résumé, la variation thermique du champ moléculaire due à la dilatation joue un rôle important dans l'interprétation des propriétés magnétiques des ferrites et très probablement aussi des anti- ferromagnétiques, notamment en ce qui concerne la comparaison du point de Curie asymptotique et du point de transition dont les valeurs relatives sont altérées.

Remarque de M. Fö̈x. - La correction de dilatation ne se manifeste pas dans le cas du nickel au-dessus de son point de Curie. La constante de Curie du nickel, qui possède un point de Curie assez élevé, est identique à celle du palladium et du platine pour lesquels les points de Curie sont négatifs. Si la dilatation intervenait elle devrait être nettement différente.

Réponse de M. Néel. - Pour ma part, j'estime que l'identité des constantes de Curie du nickel, du palladium et du platine n'a pas de signification fondamentale.

Remarque de M. Casimir. - Il serait intéressant d'étudier aussi l'influence de la pression sur les propriétés magnétiques des ferrites. Est-ce qu'on a déjà fait de telles mesures ?

Réponse de $M$. Néel. - Pas à ma connaissance.

BIBLIOGRAPHIE.

[1] Fö̈x G. el Serres A. - C. R. Acad. Sc., r $950,230,729$. [2] Maroni P. - Colloques de Ferromagnétisme et d'Antiferromaguétisme, Grenoble, I'9 5o ( $J$. Phys., i 95 г , 12, 256).

[3] NéEl L. - Ann. Phys., i 948, 3, г 37.

[4] Endô K. - Sc. Rep. Tôhoku Imp. Univ., r 937 , 25, 879.

[5] Néel L. - Ann. Inst. Fourier, I 949, 1, i 63.
[6] Néel L. - Ann. Phys., i937, 8, 237.

[7] Pour les notations, se reporter aux références [3] ou [5].

[8] Fowler R. H. et Guggenheim E. A. - Statistical Thermodynamics, Cambridge, 1939 .

[9] Chevenard P. - C. R. Acad. Sc., r 92 I , 172, 320. [10] R. Pethaunet. - C. R. Acad. Sc., i 950,230, i 842. 